\title{
Efficient multi-descriptor fusion for non-intrusive appliance recognition
}

\author{
Yassine Himeur, Abdullah Alsalemi, Faycal Bensaali \\ Department of Electrical Engineering, \\ Qatar University, Doha, Qatar \\ Email: yassine.himeur@qu.edu.qa, a.alsalemi@qu.edu.qa, \\ f.bensaali@qu.edu.qa
}

\author{
Abbes Amira \\ Institute of Artificial Intelligence, \\ De Montfort University, Leicester, United Kingdom \\ Email: abbes.amira@dmu.ac.uk
}

\begin{abstract}
Consciousness about power consumption at the appliance level can assist user in promoting energy efficiency in households. In this paper, a superior non-intrusive appliance recognition method that can provide particular consumption footprints of each appliance is proposed. Electrical devices are well recognized by the combination of different descriptors via the following steps: (a) investigating the applicability along with performance comparability of several time-domain (TD) feature extraction schemes; (b) exploring their complementary features; and (c) making use of a new design of the ensemble bagging tree (EBT) classifier. Consequently, a powerful feature extraction technique based on the fusion of TD features is proposed, namely fTDF, aimed at improving the feature discrimination ability and optimizing the recognition task. An extensive experimental performance assessment is performed on two different datasets called the GREEND and WITHED, where power consumption signatures were gathered at $1 \mathrm{~Hz}$ and $44000 \mathrm{~Hz}$ sampling frequencies, respectively. The obtained results revealed prime efficiency of the proposed fTDF based EBT system in comparison with other TD descriptors and machine learning classifiers.
\end{abstract}

Index Terms-Appliance recognition, time-domain descriptors, feature extraction, classification, fusion, ensemble bagging tree.

\section{INTRODUCTION}

Energy saving and the reduction of carbon emission are progressively considered as priorities, not only for individual users, but for decision makers as well [1], [2], [3]. To that end, research on non-intrusive load monitoring (NILM) and appliance recognition [4], [5], [6] have been investigated to collect specific appliance consumption statistics at low cost without a request for implementing individual sub-meters. In that respect, capturing individual device energy usage statistics can help detect energy-hungry appliances, biggest energy wasters and anomalous consumption [7], [8]. Thereby, we can enhance energy demand management and reduce wasted electricity via generating appropriate recommendations for individual users [9], [10], [11].

Several characteristic extraction descriptors have been investigated in the state-of-the-art literature. These comprise harmonics and transient features (HTF) [12], spectral decomposition [13], correlation-based power analytics [14], feature based events detection [15], [16], power peaks detection [17], wavelet analysis [18], [19] and voltage-current trajectory (VI)[20]. In addition, a comparison of 36 feature descriptors that were explored for appliance recognition is illustrated in
[21]. In spite of these efforts, developments of robust and efficient non-intrusive appliance recognition systems that can be easily used for in-home energy monitoring, are perceptibly confined. Further, research in that respect, still exposes a gap between academics and industry requirements. Many factors are responsible for this difference including poor recognition accuracy, variability of data from a region to another and the lack of robustness against interferences generated through the measurement campaign [22], [23].

In this paper, we propose a simple yet effective multidescriptor fusion that not only provide high performances in device recognition but also support a real-time implementation as it is based on the joint use of various time-domain (TD) feature extraction schemes, which requires significantly lower computational complexity than time-frequency analysis. Accordingly, the proposed fusion of TD features denoted as fTDF (fusion of TD features) can not only derive characteristic profiles from the current segmentation windows, but also combines those with features collected from previous windows to form more efficient appliance recognition. In this respect, the regional variation of the power consumption properties is correlated to the semi-global variation as drawn off using the TD analysis of the past windows. Moreover, in view of achieving a high accuracy, an ensemble bagging tree (EBT) classifier is designed and its performance is then compared in terms of the accuracy and F-score with various other classification models.

\section{PRoposed SySTEM}

A typical block diagram of a non-intrusive appliance recognition system is illustrated in Fig. 1].

\section{A. Feature extraction}

With a view to describe the TD feature descriptors used under our framework, let us consider a sampled version of the energy consumption signal denominated as: $s[i]$, with $i=1,2, \cdots, M$, of length $M$ and collected at the sampling frequency $f_{s}$, in order to extract the TD features, a windowing process is applied on $s$ where a window length $N$ is utilized and the TD property $S(k)$ of each window $k(k=1,2, \cdots, K$ and $K$ is the number of extracted windows) is then collected as follows: 


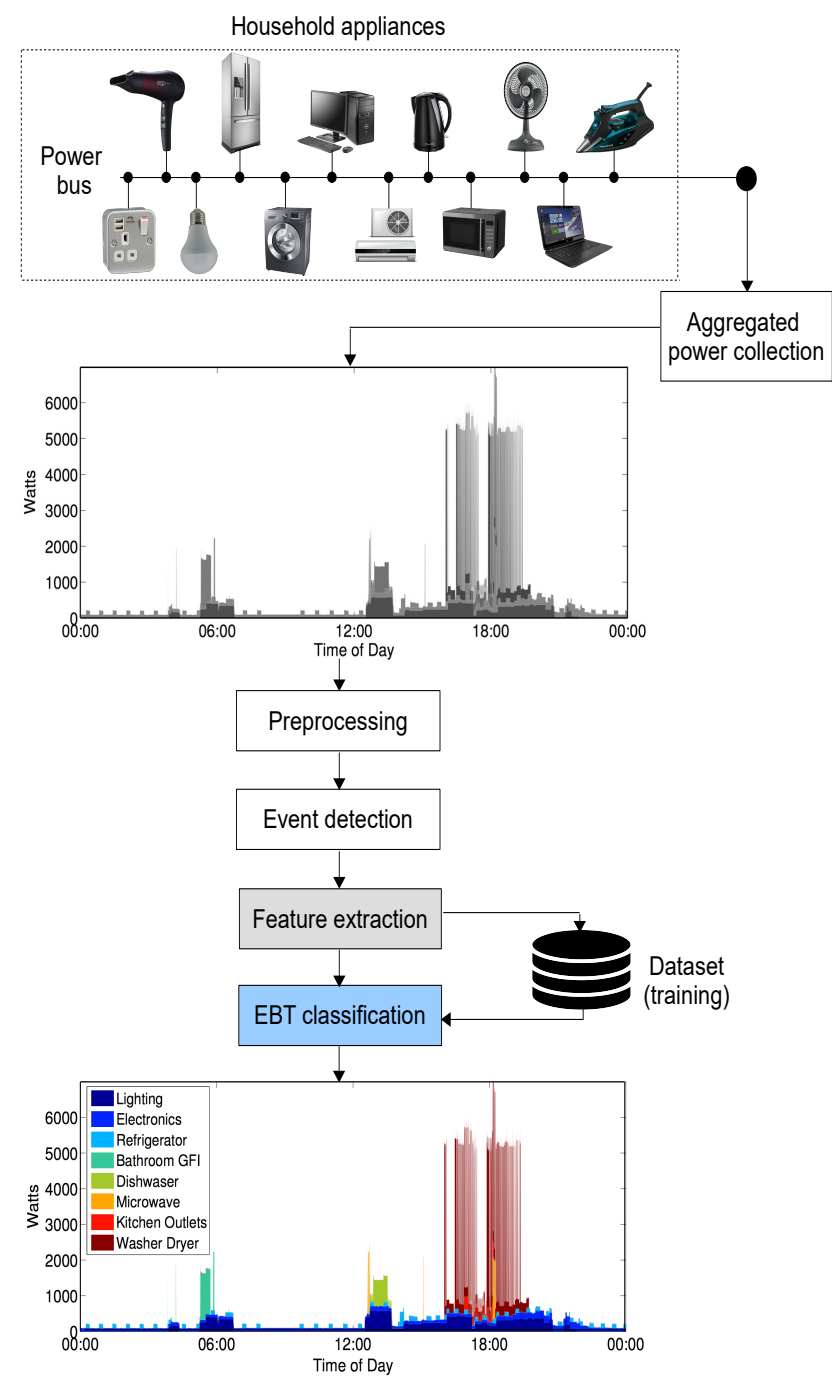

Fig. 1: Block diagram of the proposed appliance recognition system.

- Root mean square feature (RMSF)

$$
S_{R M S}(k)=\sum_{i=1}^{N} \sqrt{\frac{1}{N}\left(s_{i}^{2}\right)}
$$

- Mean absolute deviation feature (MADF)

$$
S_{M A D}(k)=\sum_{i=1}^{N} \frac{1}{N}\left|s_{i}-\mu\right|
$$

where $\mu$ represent the central tendency,

- Integrated absolute magnitude feature (IAMF)

$$
S_{\text {IAMF }}(k)=\frac{1}{N} \sum_{i=1}^{N} \frac{s_{i}^{2}}{2} \operatorname{sgn}\left(s_{i}\right)+\mu
$$

- Zero crossing feature (ZCF)

$$
S_{Z C}(k)=\sum_{i=2}^{N}\left|\operatorname{sgn}\left(s_{i}\right)-\operatorname{sgn}\left(s_{i-1}\right)\right|
$$

- Waveform length feature (WLF)

$$
S_{W L}(k)=\log \left(\sum_{i=1}^{N-1}\left|s_{i+1}-s_{i}\right|\right)=\log \left(\sum_{i=1}^{N-1}\left|\Delta s_{i}\right|\right)
$$

- Slope sign change feature (SSCF)

$$
S_{S S C}(k)=\sum_{i=2}^{N-1} f\left[\left(s_{i}-s_{i-1}\right) \times\left(s_{i}-s_{i+1}\right)\right]
$$

where

$$
f(s)= \begin{cases}1 & \text { if } s \geq \text { threshold } \\ 0 & \text { otherwise }\end{cases}
$$

- Auto-regressive feature (ARF)

$$
S_{A R F}(k)=\sum_{i=1}^{N}\left(\sum_{p=1}^{P} a_{p} s_{i-p}+w_{i}\right)
$$

where $s_{i-p}$ are the previous samples of the power consumption signal, $w_{i}$ is a white noise and $P$ represents the auto-regressive (AR) model order. Under this framework, $P=15$ is considered.

After extracting the features $S(k), k=1,2, \cdots, K$ from the different windows using an overlapping process, these data are concatenated to form the whole feature vector $S_{F}=$ $[S(1) S(2), \cdots, S(K)]$ of each TD descriptor.

\section{B. Fusion of TD features (fTDF)}

As a second contribution, a data fusion strategy named fTDF is developed, in which the characteristic vector chosen from the current window is fused with the characteristic vectors derived from the $n t h$ previous window using four different TD descriptors. In this respect, if the previous characteristic window is from the same appliance category, then the correlation rate will increase. On the flipside, for dissimilar appliance groups, the correlation rate will diminish. In addition, following the same process, we can correlate with the $2 r d, 3 r d$, and other window vectors as well. This experiment demonstrated that not only the first previous windows can be used for correlation, but further apart windows can also be considered ( e.g., the $3 t h$ previous window vector can be a good alternative since there is no performance improvement after that point). The flowchart of the proposed fTDF approach is depicted in Fig. 2, where the variation of the energy consumption data at each window is correlated to the semi-global variation of the past windows using two different TD descriptors TDF1 and TDF2. The same process is repeated using two other TD descriptors TDF3 and TDF4. Then, the correlation obtained from both scenarios is fused using a multiplication process to form the novel feature vector.

\section{Ensemble bagging tree (EBT) classifier}

The EBT model is a powerful classifier that did not receive its merit in practice. The importance of EBT comes from the fact that it can achieve a high classification performance by using a fusion of various weak classifiers. If we consider a prediction function $g(y, K)$ for predicting a category label 


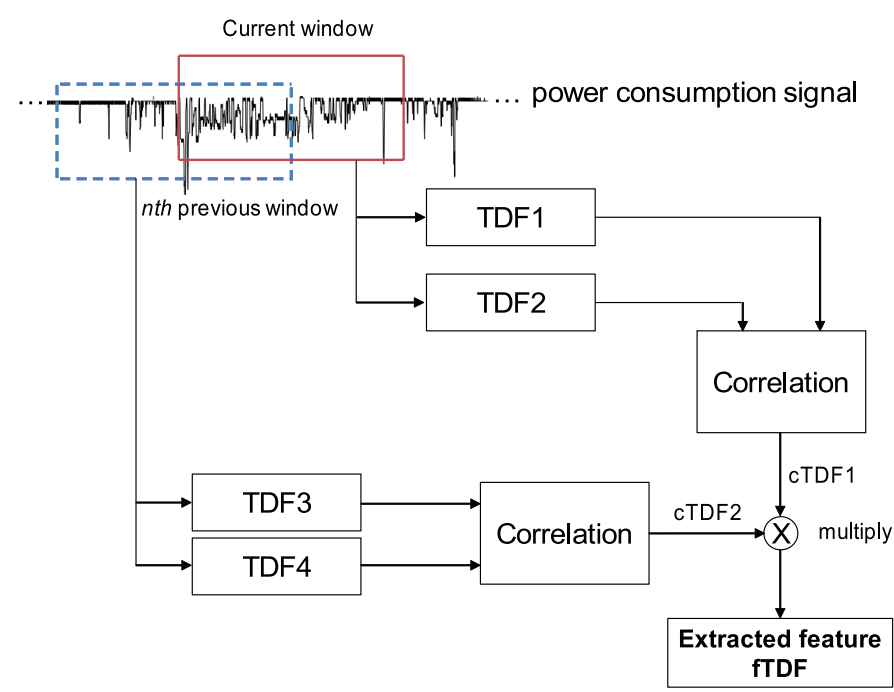

Fig. 2: Block diagram of the proposed feature extraction based data fusion architecture.

$c \in\{1,2,3 \ldots C$, where $C$ represents the whole number of categories).

$$
q(c \mid y)=P(g(y, K)=c)
$$

and the whole probability of correct classification can be given as

$$
w_{c}=\left[\sum_{c} q(c \mid y) P(c \mid y)\right] P_{y} d_{y}
$$

where $q(c \mid y)$ represents the conditional probability for the forecasted group, hence, the total accuracy can be reached using the formula of Eq. $10, P y(y)$ outlines the probability distribution of y. In addition, if $O$ is the original set of all inputs $y$ where $\varphi$ is order-correct, the correct classification rate can be estimated as

$$
\begin{gathered}
w_{A}=\int_{y \in O} \max P(c \mid y) P y(y) \\
+\int_{y \in O^{\prime}}\left[\sum_{c} I\left(\varphi_{A}(y)=c\right) P(c \mid y) P y(y)\right]
\end{gathered}
$$

where $I(\cdot)$ represents the indicator function. From Eq. 11 it can be drawn that for an order-correct $\varphi$ for the inputs $y$, the accurate classification ratio is a long way of being optimal, while $\varphi_{A}$ is optimal. Moreover, we can also deduce that even if a classifier is good (i.e., order-correct for nearly all the inputs $y$ ), it can be considered nearly optimal when it is constituted of multiple weak classifiers.

\section{EXPERIMENTAL RESULTS}

\section{A. Datasets description}

Two datasets are used in this framework to validate the proposed appliance recognition system. They are the GREEND [24] and WHITED [25] collected at low and high frequency sampling rates, respectively. The WHITED includes the power consumption signatures of the device start-ups collected from
TABLE I: Description of monitored appliances on both the WHITED and GREEND datasets

\begin{tabular}{llr|llr}
\hline \multicolumn{2}{c|}{ WHITED } & \multicolumn{3}{c}{ GREEND } \\
\hline Tag & $\begin{array}{l}\text { Apliance } \\
\text { category }\end{array}$ & $\begin{array}{l}\text { \# mesured } \\
\text { apliances }\end{array}$ & Tag & $\begin{array}{l}\text { Appliance } \\
\text { Category }\end{array}$ & $\begin{array}{r}\text { \# mesured } \\
\text { days }\end{array}$ \\
\hline 1 & Modem/receiver & 20 & 1 & Coffee machine & 242 \\
2 & CFL & 20 & 2 & Radio & 242 \\
3 & Charger & 20 & 3 & Fridge / freezer & 240 \\
4 & Coffee machine & 20 & 5 & Dishwasher & 242 \\
5 & Drilling machine & 30 & 6 & TV & 242 \\
6 & Fan & 20 & & & 242 \\
7 & Flatron & 20 & & & \\
8 & LED ight & 20 & & & \\
9 & Kettle & 20 & & & \\
10 & Microwave & 20 & & & \\
11 & Iron & & & & \\
\hline
\end{tabular}

up 110 electrical devices, categorizing up to 47 appliance groups. For each appliance category, a set of power consumption fingerprints is collected from different appliance manufacturers and all of them are gathered at a sampling rate of $44000 \mathrm{~Hz}$. In this framework, we use 11 appliance categories to validate the proposed system. The GREEND contains the electricity consumption signatures of several domestic appliances collected at a sampling frequency of 1 $\mathrm{Hz}$ from 8 households in Italy and Austria. To validate the proposed system, we use energy usage footprints gathered from typical house. Table 1 depicts the electrical devices used to collect load consumption signatures and the monitoring period for both the WHITED and GREEND.

MATLAB 2018a is employed to carry out the analysis. A segmentation based on an overlapping rate of $1 / 4$ the window size is utilized for all the descriptors during this study. The proposed fTDF approach is designed using the fusion of four TD decriptors, including MADV, IAVF, RMSF and WLF. With a view of evaluating the performance of the proposed fTDF, the obtained results' accuracy (acc) and F-score (F-scr) are compared to those extracted from the various TD descriptors described in section II-A

Fig. 3 and Fig. 4 portray the comparison of the accuracy and F-score performances of fTDF against the other TD feature extraction schemes, according to a window size ranging from 64-4096 for both the GREEND and WHITED datasets. The obtained results show the superiority of the proposed fTDF in both cases. The best performances can be obtained for a window length of 3072 . This can be justified by the fact that through aggregating several weak descriptors using the proposed fusion architecture, a powerful descriptor is designed that can improve feature discrimination ability and thus optimize the classification process. Therefore, this is the main advantage of the this fusion process. Furthermore, the slight difference between the results collected from the WHITED and GREEND is due to the fact that these datasets are quite different. The first collects the power consumption signatures of each device for a very short duration $(5 \mathrm{sec})$ while the second one gathers energy usage footprints of each appliance for the whole day. 

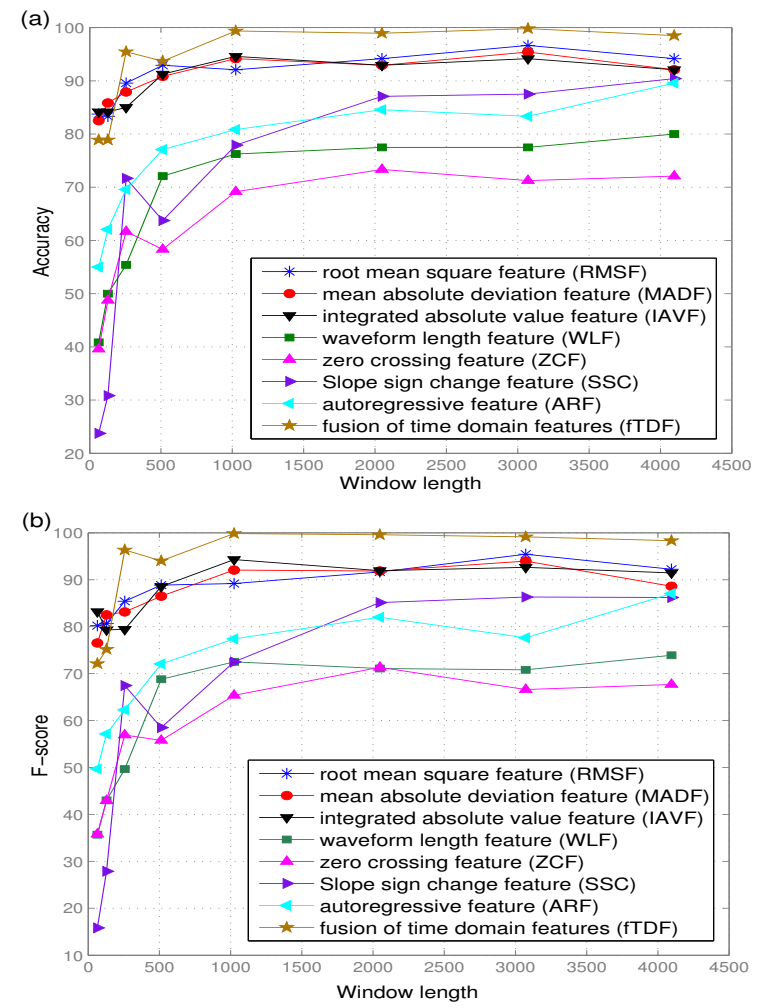

Fig. 3: Performances variation according to the window length for the WHITED: a) accuracy and b) F-score.

\section{B. Comparison with other classifiers}

We perform a performance comparison of the EBT classifier versus other machine learning models including support vector machine (SVM), deep neural networks (DNN), K-nearest neighbors $(\mathrm{KNN})$, decision tree (DT) operating by reference to different classification parameters and when the fTDF is considered as well. Table II illustrates the accuracy and Fscore outputs obtained for both the GREEND and WHITED datasets where a window length of 3072 is considered. It is clearly shown that the EBT outperformed the other classifiers with respect to the accuracy and F-score.

\section{CONCLUSION}

In this framework, a robust non-intrusive appliance system based on a multi-descriptor fusion was proposed. First, various TD descriptors were explored individually to verify their corresponding performances. Second, based on the obtained results, a multiple TD feature fusion technique denoted as fTDF was introduced. In this proposed technique, the correlation vectors extracted from four TD descriptors using an improved windowing process were then combined. This helped mitigating the limitation of each one and also improving overall feature discrimination ability. Third, the new EBT design was introduced to classify the fTDF patterns extracted from two realistic datasets. The attained results showed improved results compared to various TD descriptors and other classifiers. Future works will aim to analyze appliance specific data collected
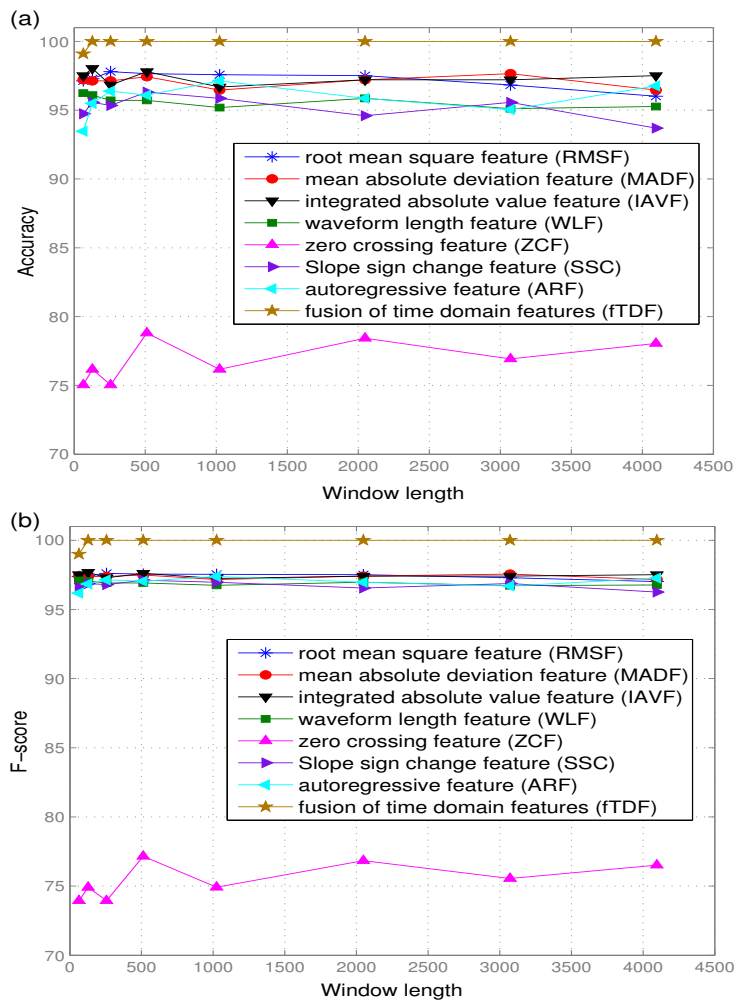

Fig. 4: Performances variation according to the window length for the GREEND: a) accuracy and b) F-score.

TABLE II: Accuracy and F-score of the EBT model compared with other classifiers using fTDF

\begin{tabular}{l|c|c|c|c|c}
\hline \multirow{2}{*}{$\begin{array}{l}\text { ML } \\
\text { classifier }\end{array}$} & \multirow{2}{*}{$\begin{array}{c}\text { Classifier } \\
\text { parameters }\end{array}$} & \multicolumn{2}{c|}{ GREEND } & \multicolumn{2}{c}{ WHITED } \\
\cline { 3 - 6 } SVM & Linear Kernel & 83.41 & 82.74 & 96.5 & 96.14 \\
\hline SVM & Quadratic kernel & 77.45 & 72.67 & 97.16 & 97.3 \\
\hline SVM & Gaussian kernel & 83.86 & 82.99 & 88.75 & 85.75 \\
\hline KNN & K=1, Euclidean dist & 96.53 & 96.54 & 83.75 & 78.77 \\
\hline KNN & $\begin{array}{c}\text { K=10, Weighted } \\
\text { Euclidean dist }\end{array}$ & 95.78 & 95.77 & 82.91 & 76.8 \\
& K=10, Cosine dist & 93.13 & 93.09 & 81.25 & 74.13 \\
\hline KNN & Fine, 100 splits & 93.96 & 93.95 & 81.25 & 77.93 \\
\hline DT & Medium, 20 splits & 94.64 & 94.66 & 82.5 & 79.7 \\
\hline DT & Coarse, 4 splits & 62.89 & 53.81 & 39.58 & 28.94 \\
\hline DT & 50 hidden layers & 97.11 & 96.93 & 96.87 & 96.61 \\
\hline DNN & 30 learners, 42 k & $\mathbf{1 0 0}$ & $\mathbf{1 0 0}$ & $\mathbf{9 9 . 6 9}$ & $\mathbf{9 9 . 4 7}$ \\
\hline EBT & splits & & & & \\
\hline
\end{tabular}

from the fTDF based EBT system, detect abnormal usage and then generate appropriate recommendations to enhance energy efficiency.

\section{ACKNOWLEDGEMENTS}

This paper was made possible by National Priorities Research Program (NPRP) grant No. 10-0130-170288 from the Qatar National Research Fund (a member of Qatar Foundation). The statements made herein are solely the responsibility of the authors. 


\section{REFERENCES}

[1] K. He, L. Stankovic, J. Liao, and V. Stankovic, "Non-intrusive load disaggregation using graph signal processing," IEEE Transactions on Smart Grid, vol. 9, no. 3, pp. 1739-1747, May 2018.

[2] A. Alsalemi, C. Sardianos, F. Bensaali, I. Varlamis, A. Amira, and G. Dimitrakopoulos, "The role of micro-moments: A survey of habitual behavior change and recommender systems for energy saving," IEEE Systems Journal, vol. 13, no. 3, pp. 3376-3387, Sep. 2019.

[3] M. A. Devlin and B. P. Hayes, "Non-intrusive load monitoring and classification of activities of daily living using residential smart meter data," IEEE Transactions on Consumer Electronics, vol. 65, no. 3, pp. 339-348, Aug 2019.

[4] S. Welikala, C. Dinesh, M. P. B. Ekanayake, R. I. Godaliyadda, and J. Ekanayake, "Incorporating appliance usage patterns for non-intrusive load monitoring and load forecasting," IEEE Transactions on Smart Grid, vol. 10, no. 1, pp. 448-461, Jan 2019.

[5] V. Andrean, X. Zhao, D. F. Teshome, T. Huang, and K. Lian, "A hybrid method of cascade-filtering and committee decision mechanism for nonintrusive load monitoring," IEEE Access, vol. 6, pp. 41212-41223, 2018.

[6] Q. Liu, K. M. Kamoto, X. Liu, M. Sun, and N. Linge, "Low-complexity non-intrusive load monitoring using unsupervised learning and generalized appliance models," IEEE Transactions on Consumer Electronics, vol. 65, no. 1, pp. 28-37, Feb 2019.

[7] D. Li and S. Dick, "Residential household non-intrusive load monitoring via graph-based multi-label semi-supervised learning," IEEE Transactions on Smart Grid, vol. 10, no. 4, pp. 4615-4627, July 2019.

[8] M. Gaur and A. Majumdar, "Disaggregating transform learning for nonintrusive load monitoring," IEEE Access, vol. 6, pp. 46256-46265, 2018.

[9] A. Alsalemi, M. Ramadan, F. Bensaali, A. Amira, C. Sardianos, I. Varlamis, and G. Dimitrakopoulos, "Endorsing domestic energy saving behavior using micro-moment classification," Applied Energy, vol. 250, pp. $1302-1311,2019$.

[10] A. Alsalemi, F. Bensaali, A. Amira, N. Fetais, C. Sardianos, and I. Varlamis, "Smart energy usage and visualization based on micromoments," in Intelligent Systems and Applications, Y. Bi, R. Bhatia, and S. Kapoor, Eds. Cham: Springer International Publishing, 2020, pp. $557-566$.

[11] C. Sardinos, I. Varlamis, G. Dimitrakopoulos, A. Alsalemi, F. Bensaali, and A. Amira, "I want to .... change" micro-moment based recommendations can change users," in in Proc. 8th International Conference on Smart Cities and Green ICT Systems, May 2019, pp. 1 - 10.

[12] G. W. Hart, "Nonintrusive appliance load monitoring," Proceedings of the IEEE, vol. 80, no. 12, pp. 1870-1891, Dec 1992.

[13] S. Welikala, N. Thelasingha, M. Akram, P. B. Ekanayake, R. I. Godaliyadda, and J. B. Ekanayake, "Implementation of a robust real-time non-intrusive load monitoring solution," Applied Energy, vol. 238, pp. 1519 - 1529, 2019

[14] N. Roy, N. Pathak, and A. Misra, "Fine-grained appliance usage and energy monitoring through mobile and power-line sensing," Pervasive and Mobile Computing, vol. 30, pp. 132 - 150, 2016.

[15] X. Wu, X. Han, and K. X. Liang, "Event-based non-intrusive load identification algorithm for residential loads combined with underdetermined decomposition and characteristic filtering," IET Generation, Transmission Distribution, vol. 13, no. 1, pp. 99-107, 2019.

[16] L. Wang, W. Cho, Y. Chiu, and C. Lai, "A parallel multi-appliance recognition for smart meter," in 2013 IEEE 11th International Conference on Dependable, Autonomic and Secure Computing, Dec 2013, pp. 475-480.

[17] D. Yan, Y. Jin, H. Sun, B. Dong, Z. Ye, Z. Li, and Y. Yuan, "Household appliance recognition through a bayes classification model," Sustainable Cities and Society, vol. 46, p. 101393, 2019.

[18] J. M. Gillis and W. G. Morsi, "Non-intrusive load monitoring using semisupervised machine learning and wavelet design," IEEE Transactions on Smart Grid, vol. 8, no. 6, pp. 2648-2655, Nov 2017.

[19] H. Chang, K. Lian, Y. Su, and W. Lee, "Power-spectrum-based wavelet transform for nonintrusive demand monitoring and load identification," IEEE Transactions on Industry Applications, vol. 50, no. 3, pp. 20812089, May 2014.

[20] Y. Liu, X. Wang, and W. You, "Non-intrusive load monitoring by voltageâĂŞcurrent trajectory enabled transfer learning," IEEE Transactions on Smart Grid, vol. 10, no. 5, pp. 5609-5619, Sep. 2019.
[21] M. Kahl, A. Ul Haq, T. Kriechbaumer, and H.-A. Jacobsen, "A comprehensive feature study for appliance recognition on high frequency energy data," in Proceedings of the Eighth International Conference on Future Energy Systems, ser. e-Energy '17. New York, NY, USA: ACM, 2017, pp. 121-131.

[22] H. N. Rafsanjani and A. Ghahramani, "Towards utilizing internet of things (iot) devices for understanding individual occupants' energy usage of personal and shared appliances in office buildings," Journal of Building Engineering, vol. 27, p. 100948, 2020.

[23] Y. Chen, T. Hong, X. Luo, and B. Hooper, "Development of city buildings dataset for urban building energy modeling," Energy and Buildings, vol. 183, pp. 252 - 265, 2019.

[24] A. Monacchi, D. Egarter, W. Elmenreich, S. D'Alessandro, and A. M. Tonello, "Greend: An energy consumption dataset of households in italy and austria," in 2014 IEEE International Conference on Smart Grid Communications (SmartGridComm), Nov 2014, pp. 511-516.

[25] M. Kahl, A. U. Haq, T. Kriechbaumer, and H.-A. Jacobsen, "Whiteda worldwide household and industry transient energy data set," in $3 r d$ International Workshop on Non-Intrusive Load Monitoring, 2016. 